\author{
Original Article
}

\title{
Evaluation of Maxillofacial Characteristics in Individuals with Anterior Open Bite Using Denture Frame Analysis
}

Emre Cesur' iD, Ayşegül Köklü

\begin{abstract}
'Department of Orthodontics, Istanbul Medipol University Faculty of Dentistry, Istanbul, Turkey
Department of Orthodontics, Ankara University Faculty of Dentistry, Ankara, Turkey

Cite this article as: Cesur E, Köklü A. Evaluation of Maxillofacial Characteristics in Individuals with Anterior Open Bite Using Denture Frame Analysis. Turk J Orthod 2020; 33(3): 157-64.
\end{abstract}

\section{ABSTRACT}

Keywords: The aim of this study was to evaluate the effectiveness of denture frame analysis (DFA) in individuals with anterior open bite who had completed pubertal peak growth (post-peak).

Methods: This retrospective study was conducted using the cephalometric radiographs and hand-wrist X-rays of 50 individuals with open bite (mean age: 17.33 \pm 3 years; 35 female, 15 male) and a control group of 50 individuals without open bite (mean age: $17.38 \pm 2.72 ; 35$ female, 15 male). All individuals included in the study were skeletal Class I and had completed or nearly completed skeletal growth. Skeletal and dental measurements pertaining to DFA were done and the data were analyzed using independent samples $t$ test and Mann-Whitney $\mathrm{U}$ test.

Results: Measurements assessing the vertical dimension showed that GoGn/SN, Frankfurt horizontal (FH)/mandibular plane (MP), palatal plane (PP)/MP, occlusal plane (OP)/MP, and OP-MP/PP-MP measurements were significantly greater in the open bite group $(p<0.001)$. AB/MP angle was significantly larger in the control group $(p<0.05)$. The open bite group had shorter $A^{\prime}-P^{\prime}$ (posterior maxillary length; $p<0.05)$ and, therefore, higher $A^{\prime}-6{ }^{\prime} / A^{\prime}-P^{\prime}$ ratio $(p<0.01)$.

Conclusion: Our results suggest that there may be a close association between maxillary OP inclination and mandibular position in individuals with open bite, and that open bite may arise due to maxillary denture base deficiency, especially in the posterior region. DFA may be useful in the differential diagnosis of open bite and in treatment planning, particularly when determining the need for tooth extraction.

Keywords: Denture frame analysis, occlusal plane, open bite, posterior discrepancy

Main points:

- Posterior arch length deficiency, particularly at the distal of the first molars can be present in individuals with open bite.

- Open bite may arise due to maxillary denture base deficiency.

- Mandibular position can be affected by the changes in the occlusal plane.

\section{INTRODUCTION}

Craniofacial growth estimation is important in orthodontics both in terms of diagnosis and treatment. Condylar growth has long been considered the primary factor affecting mandibular growth (1, 2). However, some studies suggest that condylar adaptation may arise as a result of mandibular repositioning and that changes in the position of the mandible may affect condylar growth $(3,4)$.

Cephalometric analyses usually examine the relationship of teeth with the cranial base and its effects on skeletal and soft tissue. In such skeletal and dental analyses, it is generally accepted that the impaired relationship between the maxilla and the mandible is caused by undesired growth pattern. However, the exact cause of excessive mandibular growth has not been established. It is still unclear whether the growth in condylar region is a result of genetic or adaptive effects. According to the functional matrix theory, skeletal units (i.e., the bony 
structures that support and protect operational functional units) grow and adapt according to changes that occur in functional units (5-7).

Petrovic (8) investigated the growth of maxillofacial skeletal structures and described the "cybernetic model" based on Moss's concept. According to this model, mandibular position is largely affected by the functional needs, particularly articulation, and this is regulated by the central musculoskeletal system. Occlusal function is an important factor for mandibular growth. The displacement of the maxilla through forward and downward directs the growth of the mandible, and the mandible tries to adapt to this displacement functionally. In addition, the temporomandibular joint adapts to the new position of the mandible. According to the cybernetic theory, the functional factor regulating the mandibular growth is occlusal function. For example, functional occlusal planes (OPs) of an individual whose maxilla is developing downward will be located below and in response to this, the mandible will move vertically and develop in a vertical direction. The functional forces of the mandible will be transmitted through the temporal bone with the help of the temporomandibular joint and the masseter muscle, and movements or rotations will occur in the temporal bone. In addition, by changing the position of the mandible, the tensions of the lateral and medial pterygoid muscles change, causing the movement of sphenoid bone. The movement in the sphenoid bone changes the motion of the maxilla and affects the vertical position of the vomer. In short, the balance of the maxillofacial skeleton is affected by adapting the mandible to an abnormal occlusion with an abnormal growth pattern (9).

On the basis of this theory, Sato (10) claimed that the growth and morphology of the face was affected by the function of the occlusion and the OP. According to Sato et al. (11), the vertical positions of the posterior teeth do not stay stable during growth, especially in patients with open bite and/or high angle Class III. The OP is largely affected by its relationship with denture base deficiency posterior to the first molar. Posterior discrepancy results in the eruption of the molars during both the growth and post-pubertal period, which can also result in abnormal mandibular position and growth. The relationship of sagittal and vertical skeletal components with the occlusion should be taken into consideration while revealing the etiology of the malocclusion. Therefore, denture frame analysis (DFA) was introduced in 1987 by Sato. DFA is an easy diagnostic indicator that examines the structure of the lower face, including the skeletal frame of dentition, vertical component of the etiological factor, OP, and posterior insufficiency. It enables assessment and analysis of the vertical and sagittal relationship between the jaws and their associated OPs. DFA does not use the cranial reference planes, but is limited to the jaws and the teeth. Moreover, most cephalometric analyses based on cranial reference planes analyze vertical and sagittal relationships but overlook the fact that these two directions will affect one another (12-14). However, DFA allows evaluation of the relationships between facial type and OP, between anteroposterior problem and vertical component, and between changes in vertical dimensions and posterior insufficiency (15).
It is very difficult to control the vertical component of malocclusions. The most widely accepted and commonly used reference measurement in the evaluation of this component is the mandibular plane (MP) angle. However, the correlation between the MP angle and the vertical problem is not always sufficient for diagnosis and treatment planning (10). In their study, evaluating the norms of DFA in the Turkish population, Kayasu and Koklu (16) noted that their results differed from those obtained in the Japanese population and reported that differences may be observed based on sex and stage of skeletal maturity. Their results also demonstrated that DFA is very effective in establishing a differential diagnosis in the evaluation of the vertical dimension.

Therefore, the aim of this study was to evaluate the effectiveness of DFA as an adjunct to routinely used cephalometric analyses in order to enable occlusion-oriented evaluation for diagnosis, differential diagnosis, growth estimation, prognosis, and treatment planning in individuals with open bite who completed pubertal peak growth (post-peak). The null hypothesis of this study was "no difference exists between DFA measurements of individuals with and without open bite."

\section{METHODS}

We used GPower 3.1.0 software package (Universität Düsseldorf, Düsseldorf, Germany) to determine the number of individuals included in the study, and we further performed a power analysis. Based on a previous study, an expected effect size of $f=0.56$ was used in the power calculation (15). Sample size calculation was based on the ability to detect significant differences in $A^{\prime}-6^{\prime}$ and $A^{\prime}-P^{\prime}$ values at $a=0.05$ error probability (critical t: 1.664125 ; noncentrality parameter $\delta: 2.535508$ ). According to power analysis, a sample size of 41 patients for each group would allow for a power $>80 \%$ (actual power: 0.807608 ) with an allocation ratio (N2/N1) $=1$.

Therefore, we analyzed the cephalometric radiographs and hand-wrist X-rays of 50 individuals with open bite (mean age: $17.33 \pm 3$ years; 35 female, 15 male) and 50 individuals comprising the control group (mean age: 17.38 $\pm 2.72 ; 35$ female, 15 male) who presented to the Dentistry Hospital of the İstanbul Medipol University Mega Hospitals Complex and to the Ankara University Faculty of Dentistry for examination or treatment. The study was approved by the İstanbul Medipol University Ethics Committee (Approval No: 639) and conducted according to the principles set in the Declaration of Helsinki and informed consent was obtained from the patients.

Patients were included in the open bite group based on the following criteria:

1. Anterior open bite evident in patient photographs/clinical examination (overbite $<0 \mathrm{~mm}$ ).

2. Skeletal Class I $\left(0^{\circ} \leq \mathrm{ANB} \leq 4^{\circ}\right)$ relationship, to rule out the anteroposterior differences.

3. Complete or near-complete skeletal development based on evaluation of hand-wrist radiographs (complete or near-complete fusion of radial epiphysis and diaphysis) $(17,18)$.

4. No missing teeth other than the third molars.

5. No craniofacial syndrome or congenital abnormalities. 
Criteria 2 through 5 of the study group were also valid for the control group. Meanwhile, the individuals in the control group had overbite greater than $0 \mathrm{~mm}$.

Profile distance $X$-rays of individuals included in the study were obtained using a Sirona Orthophos XG DS/Ceph X-ray device under standard conditions with teeth in maximum intercuspal position and the Frankfurt horizontal plane parallel to the ground. During imaging, the individual's sagittal plane was $155 \mathrm{~cm}$ from the X-ray source and $12.5 \mathrm{~cm}$ from the film cassette. Maximum distance between hand and $X$-ray source was used when acquiring the hand-wrist X-rays. AutoCAD 2016 (Autodesk, CA, USA) computer software was used for data analyses. Cephalometric landmarks used in the study are given in Table 1. The following standard cephalometric measurements were made (Figure 1):

1. SNA (posteroinferior angle between anterior cranial base and nasion-point $A$ line), SNB (posteroinferior angle between anterior cranial base and nasion-point $B$ line), ANB (angle between nasion-point $A$ and nasion-point $B$ lines), and GoGn/SN (angle between anterior cranial base and Go-Gn line) angles.

2. Overjet (the horizontal distance between the maxillary and mandibular incisors) and overbite (the vertical distance between the maxillary and mandibular incisors).

Measurements used in the DFA were as follows (Figure 2) $(10,11)$ :
1. Frankfurt horizontal (FH)/MP (angle between FH line [PoOr] and MP [Go-Me]), palatal plane (PP)/MP (angle between $\mathrm{PP}$ [anterior nasal spine (ANS)- posterior nasal spine (PNS)] and MP), OP/MP (angle between the maxillary OP [U1i-U6] and $M P$ ), $A B / M P$ (angle between the line connecting $A$ and $B$ points and MP), $A^{\prime}-6^{\prime}$ (anterior maxillary length; distance between point $A^{\prime}$ and point $6^{\prime}$ ), and $A^{\prime}-P^{\prime}$ (maxillary length; distance between point $A^{\prime}$ and point $P^{\prime}$ ).

2. U1i-AB (perpendicular distance from incisal point of upper incisor to $A B$ line), $U 1 / A B$ (acute angle between axis of upper incisor, U1i-U1a, and AB line), L1 i-AB (perpendicular distance from incisal point of lower incisor to $A B$ line), $L 1 / A B$ (acute angle between axis of upper incisor, L1 $\mathrm{i}-\mathrm{L} 1 \mathrm{a}$, and $A B$ line), and intermolar angle (wide angle between long axis of upper and lower first molars).

\section{Statistical Analysis}

Measurements were performed twice by the same observer four weeks apart and correlation coefficients were calculated to assess reliability of the measurements. Data obtained in the study were analyzed with The Statistical Package for Social Sciences version 21.0 software (IBM Corp.; Armonk, NY, USA). Comparisons between two groups were done using independent samples $t$-test or Mann-Whitney $U$ test in accordance with tests of normality. Level of significance was accepted as $0.05 ; \mathrm{p}<0.05$ were considered statistically significant.

Table 1. Cephalometric skeletal and dental landmarks

\section{Skeletal Landmarks}

Nasion (N)

Sella (S)

A

B

Gonion (Go)

Gnathion (Gn)

Menton (Me)

ANS

PNS

Orbitale (Or)

Porion (Po)

Pterygomaxillary fissure $(P)$

$A^{\prime}$

$P^{\prime}$

U1i

U1a

L1i

L1a

U6

U6f

L6

L6f

U6m

$6^{\prime}$
The most anterior point of the frontonasal suture

The midpoint of sella turcica

The deepest point of the concavity on the maxilla between ANS and prosthion

The deepest point of the concavity on the mandibular symphysis between infradentale and pogonion

Point of intersection of the ramus plane and the mandibular plane

The most anteroinferior point on the symphysis

The midpoint on the inferior border of the mental protuberances

The most anterior point of anterior nasal spine

The most posterior point of posterior nasal spine

The most antero-inferior point of the infraorbital rim

The most superior point of the meatus acusticus externus

The most anterior point of the pterygomaxillary fissure

Projection of point A on palatal plane (ANS-PNS line)

Projection of point $P$ on palatal plane (ANS-PNS line)

\section{Dental Landmarks}

Incisal point of upper central incisor

Apical point of upper central incisor

Incisal point of lower central incisor

Apical point of lower central incisor

The midpoint of upper first molar's occlusal surface

Furcation point between upper first molar's mesial and distal roots

The midpoint of lower first molar's occlusal surface

Furcation point between lower first molar's mesial and distal roots

The most anterior point of upper first molar crown

Projection of U6m on the palatal plane (ANS-PNS line) 


\section{RESULTS}

Repeated measurements showed high reliability, with correlation coefficients ranging between 0.882 and 0.996 .

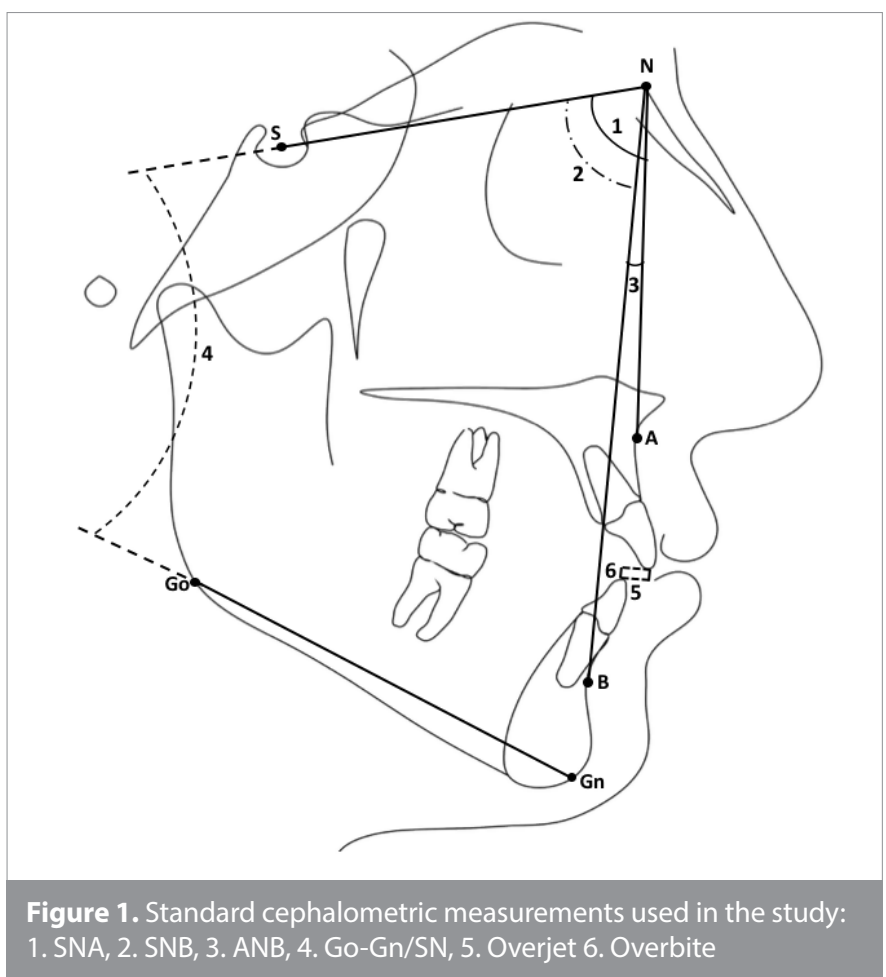

Comparison of mean values in the open bite and control group showed that the ANB angle was $2^{\circ}$ in the open bite group and $2.6^{\circ}$ in the control group $(p<0.05)$. Measurements assessing the

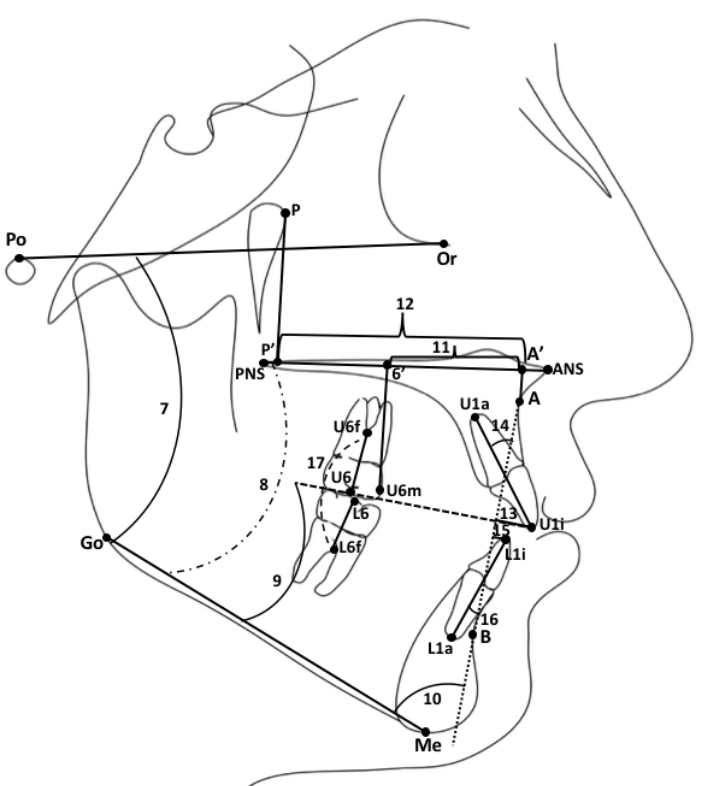

Figure 2. DFA measurements: 7. FH/MP 8. PP/MP 9. OP/MP 10. AB/ MP 11. A'-6', 12. A'-P', 13. U1i-AB, 14. U1/AB 15. L1i-AB, 16. L1/AB, 17. Intermolar angle

Table 2. Descriptive statistics and comparison of the cephalometric measurements between the open bite and control groups with $t$ test

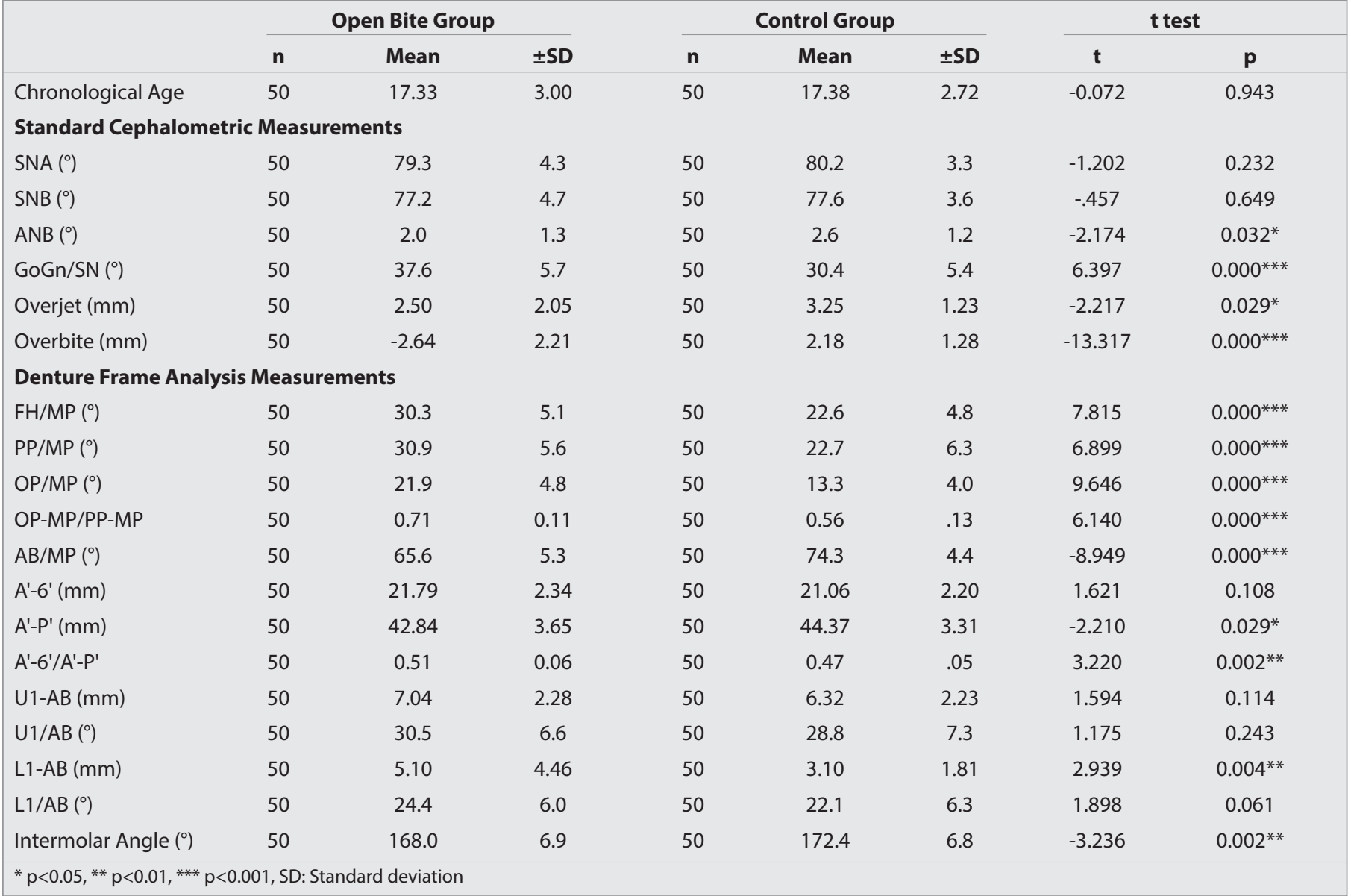


vertical dimension showed that GoGn/SN, FH/MP, PP/MP, OP/MP, and OP-MP/PP-MP measurements were significantly greater in the open bite group $(p<0.001)$. AB/MP angle was significantly larger in the control group $(p<0.05)$. The open bite group had shorter $A^{\prime}-P^{\prime}$ (maxillary length) $(p<0.05)$ and consequently higher $A^{\prime}-6^{\prime} / A^{\prime}-P^{\prime}$ ratio $(p<0.01)$. Intermolar angle, overjet, and overbite were found to be greater in the control group (Table 2).

When the groups were compared by sex, females in the open bite and control groups showed significant differences in ANB angle, GoGn/SN, FH/MP, PP/MP, OP/MP, OP-MP/PP-MP, AB/MP, $A^{\prime}-P^{\prime}, L 1-A B$, intermolar angle, overjet, and overbite, whereas males in the open bite and control groups showed significant differences in terms of ANB angle, GoGn/SN, FH/MP, PP/MP, OP/ $M P, O P-M P / P P-M P, A B / M P, A^{\prime}-6, A^{\prime}-6^{\prime} / A^{\prime}-P^{\prime}$ ratio, $L 1-A B, L 1 / A B$, intermolar angle, and overbite (Table 3 ).

According to sex-based comparisons within each group, only $A^{\prime}-P^{\prime}$ was higher in males than females in the open bite group $(p<0.05)$. In the control group, females had higher PP/MP value and $A^{\prime}-6{ }^{\prime} / A^{\prime}-P^{\prime}$ ratio compared to the males $(p<0.05$; Table 4$)$.

\section{DISCUSSION}

Although cephalometric radiographs are widely used in orthodontics to evaluate the growth and development of facial structures and treatment-induced changes in these structures, these analyses are generally inadequate to detect the change between these facial structures and the OP. According to Sato (10) the growth and morphology of the face was affected by the function of the occlusion and the OP. Citing the lack of an analysis that demonstrated the relationship between facial type and OP, between anteroposterior problem and the vertical component, or between changes in vertical dimensions and posterior deficiency in the dental arch, Sato (10) developed DFA to understand the relationship between posterior deficiency, the OP, and mandibular repositioning. Changes in the posterior OP play an important role not only in the sagittal dimension but also in the vertical dimension and position of the maxillomandibular structures (19, 20). The tooth-to-denture base discrepancy posterior to the first molar causes a "squeezing out" effect, the occlusal contacts that occur due to the crowding of the maxillary molars cause changes in mandibular position, and abnormal vertical mandibular growth may lead to open bite $(11,19)$.

On the basis of these considerations, in this study comparing OP and craniofacial morphology in individuals with and without open bite using DFA, we matched two groups in terms of chronological age and sex distribution in order to minimize intergroup differences. When selecting the study sample, we considered not only chronological age but also stage of skeletal development. It is known that normative values may be affected by growth and development and can change in later stages (21). Thus, to avoid

Table 3. Comparison of the cephalometric measurements between the open bite and control group for females and males Mann Whitney U test

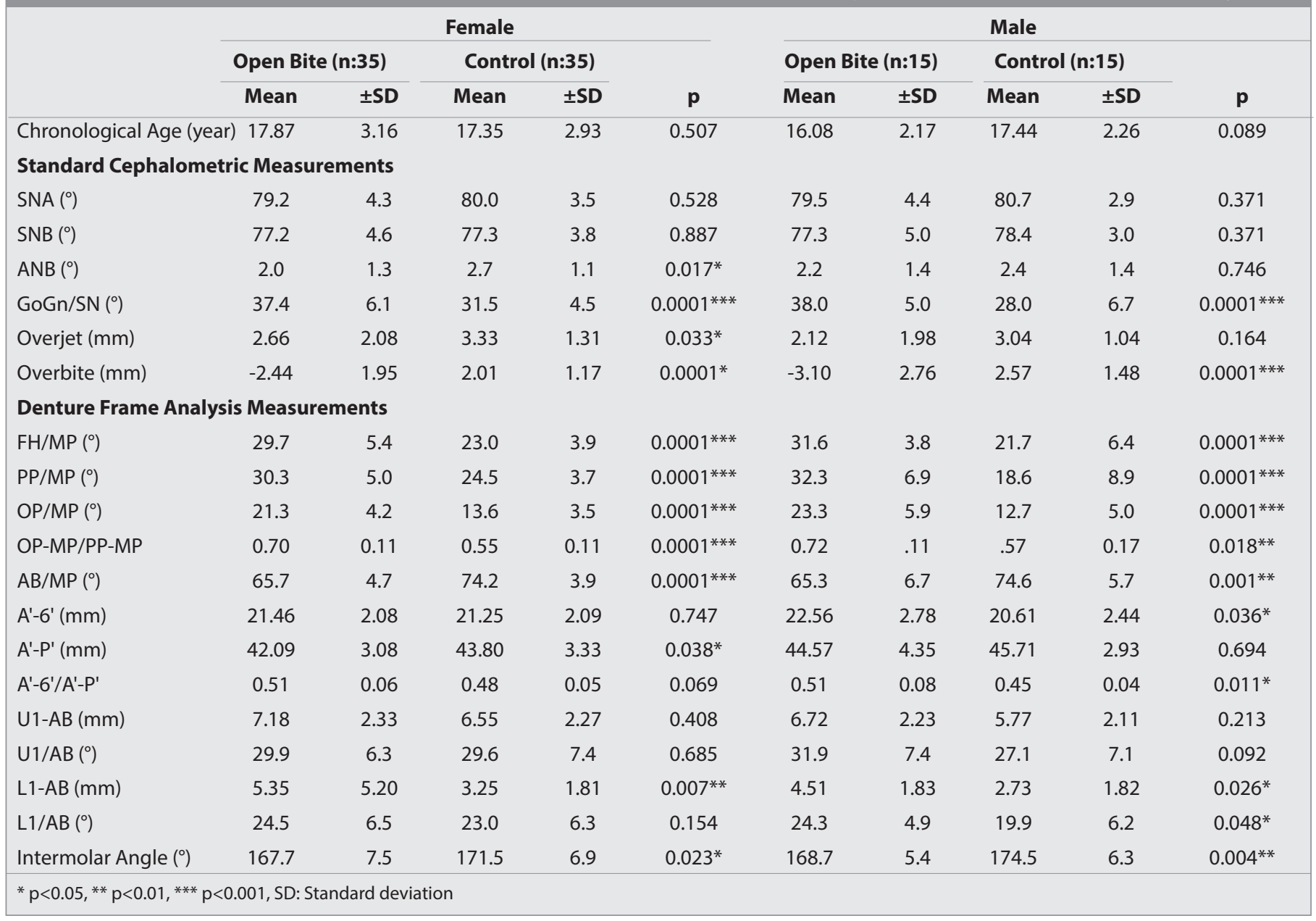


Table 4. Intragroup comparison of the cephalometric measurements between the sexes using with Mann Whitney $U$ test

\begin{tabular}{|c|c|c|c|c|c|c|c|c|c|c|}
\hline & \multicolumn{5}{|c|}{ Female } & \multicolumn{5}{|c|}{ Male } \\
\hline & \multicolumn{2}{|c|}{ Open Bite (n:35) } & \multicolumn{2}{|c|}{ Control (n:35) } & \multirow[b]{2}{*}{$\mathbf{p}$} & \multicolumn{2}{|c|}{ Open Bite (n:15) } & \multicolumn{2}{|c|}{ Control (n:15) } & \multirow[b]{2}{*}{$\mathbf{p}$} \\
\hline & Mean & \pm SD & Mean & $\pm S D$ & & Mean & \pm SD & Mean & $\pm S D$ & \\
\hline \multicolumn{11}{|c|}{ Standard Cephalometric Measurements } \\
\hline SNA $\left(^{\circ}\right)$ & 79.2 & 4.3 & 79.5 & 4.4 & 0.841 & 80.0 & 3.5 & 80.7 & 2.9 & 0.333 \\
\hline GoGn/SN $\left(^{\circ}\right)$ & 37.4 & 6.1 & 38.0 & 5.0 & 0.664 & 31.5 & 4.5 & 28.0 & 6.7 & 0.063 \\
\hline Overjet (mm) & 2.66 & 2.08 & 2.12 & 1.98 & 0.391 & 3.33 & 1.31 & 3.04 & 1.04 & 0.657 \\
\hline Overbite (mm) & -2.44 & 1.95 & -3.10 & 2.76 & 0.561 & 2.01 & 1.17 & 2.57 & 1.48 & 0.223 \\
\hline \multicolumn{11}{|c|}{ Denture Frame Analysis Measurements } \\
\hline OP-MP/PP-MP & 0.70 & 0.11 & 0.72 & 0.11 & 0.799 & 0.55 & 0.11 & 0.57 & 0.17 & 0.849 \\
\hline $\mathrm{AB} / \mathrm{MP}\left({ }^{\circ}\right)$ & 65.7 & 4.7 & 65.3 & 6.7 & 0.865 & 74.2 & 3.9 & 74.6 & 5.7 & 0.932 \\
\hline$A^{\prime}-6^{\prime}(m m)$ & 21.46 & 2.08 & 22.56 & 2.78 & 0.071 & 21.25 & 2.09 & 20.61 & 2.44 & 0.485 \\
\hline$A^{\prime}-P^{\prime}(m m)$ & 42.09 & 3.08 & 44.57 & 4.35 & $0.018^{*}$ & 43.80 & 3.33 & 45.71 & 2.93 & 0.072 \\
\hline$A^{\prime}-6{ }^{\prime} / A^{\prime}-P^{\prime}$ & 0.51 & 0.06 & 0.51 & 0.08 & 0.899 & .48 & 0.05 & 0.45 & 0.04 & $0.009^{* * *}$ \\
\hline U1-AB (mm) & 7.18 & 2.33 & 6.72 & 2.23 & 0.518 & 6.55 & 2.27 & 5.77 & 2.11 & 0.216 \\
\hline $\mathrm{U} 1 / \mathrm{AB}\left({ }^{\circ}\right)$ & 29.9 & 6.3 & 31.9 & 7.4 & 0.304 & 29.6 & 7.4 & 27.1 & 7.1 & 0.379 \\
\hline L1-AB (mm) & 5.35 & 5.20 & 4.51 & 1.83 & 0.657 & 3.25 & 1.81 & 2.73 & 1.82 & 0.285 \\
\hline $\mathrm{L} 1 / \mathrm{AB}\left(^{\circ}\right)$ & 24.5 & 6.5 & 24.3 & 4.9 & 0.815 & 23.0 & 6.3 & 19.9 & 6.2 & 0.135 \\
\hline
\end{tabular}

variation in our results related to stage of skeletal development, all individuals selected for the study had completed or nearly completed growth $(17,18)$. Although there was a significant difference between the open bite group and control group in terms of ANB angle $(p<0.05)$, we made sure to select the individuals included in both groups from among those with skeletal Class I relationship in order to limit the effect of the sagittal dimension and to better evaluate the vertical dimension (open bite group ANB: $2^{\circ}$, control group ANB: $2.6^{\circ}$ ). Consistent with ANB angle, we found that among the individuals included in our study, overjet was also slightly greater in the control group and the difference was significant between the females in the two groups $(p<0.05)$.

Skeletal open bite is not limited to the dentoalveolar region alone but includes craniofacial malformations that involve the skeletal structure and jaws $(22,23)$. In this study, the evaluation of parameters related to the vertical dimension (GoGn/SN, FH/MP, and $\mathrm{PP} / \mathrm{MP}$ ) naturally revealed significant differences between the open bite and control groups, with the open bite group displaying a larger MP angle $(p<0.001)$. Although sex-based comparisons between the groups yielded similar results, intragroup comparisons between the sexes showed that only PP/MP angle was larger in females than males in the control group, whereas no differences were detected in other measurements. In DFA, the angle between $A B$ and $M P$ is analyzed to evaluate the relationship between the maxilla and the mandible in the sagittal plane. Although this measurement provides insight into both horizontal and vertical jaw relation, Celar et al. (15) stated that this parameter alone is inadequate for the evaluation of the relationship between the maxilla and the mandible. In our study, this angle was significantly narrower in the open bite group and the difference was significant for both females and males in sex-based comparisons between the groups. We attribute this difference to the steeper MP in the open bite group rather than anteroposterior differences. AB-MP angle gives the relation of the jaws in the sagittal direction. However, as this angle also depends on the inclination of the mandibular plane, it is a measurement that evaluates the sagittal and vertical positions simultaneously. Therefore, the use of this measurement without considering vertical parameters is not appropriate for determining the anteroposterior relationship of jaws.

Occlusion and the maxillofacial structures comprise a unique and dynamic mechanism involving continuous interaction. Therefore, orthodontic treatment does not simply alter occlusion, but also changes the skeletal structures of maxillofacial structures. According to Petrovic (24), there is a direct relationship between occlusion and mandibular position. He stated that differences in the direction and amount of the condylar growth can arise in response to changes in maxillary length, and that the lower dental arch can be controlled through the continuously changing reference inputs of the upper dental arch. In DFA, the angle 
between the maxillary OP and MP demonstrates the functional adaptation capacity of the mandible, while the OP-MP/PP-MP ratio shows the ratio of the angle between MP and maxillary OP to the angle between MP and PP. Normally, in order to maintain a stable OP/MP angle, the mandible changes its position in accordance with the occlusal function. However, the mandible cannot adapt to excessive changes in the OP and undergoes posterior rotation, increasing this angle. An OP-MP/PP-MP ratio over 0.6 indicates a deviation in the OP that the mandible could not adapt to. The increase in this ratio is the suggestive of an open bite (10). The evaluation of these parameters in the present study showed that OP/MP angle was higher in the open bite group, while the OP-MP/PP-MP ratio was 0.71 , corroborating Sato's predictions. This ratio was significantly greater for both females and males in the open bite group compared to the control group. Celar et al. (15) reported an OP-MP/PP-MP ratio of 0.56 and a mean of 0.7 in open bite, whereas Sato (10) found this ratio to be 0.54 in the normal individuals. Although the increase in the angle of the MP can affect these values, the results obtained in the present study confirm these results and indeed suggest that the mandibular position can be affected by the changes in the OP.

Sato (10) stated that if there is crowding in the posterior jaw, molars will try to create room through mesialization; if this is not sufficient, the molars will sag and cause flattening of the OP. If the mandible has growth potential, it can overcome these posterior contacts with growth in the anterior aspect. If it does not have enough growth potential, open bite will occur. In DFA, $A^{\prime}-P^{\prime}$ distance is evaluated to determine the total length of the maxillary denture base, whereas anterior maxillary base length is evaluated by measuring $A^{\prime}-6$. Decrease in total maxillary length and/ or increase in $A^{\prime}-6^{\prime}$ length increases the probability of posterior deficiency. $A^{\prime}-6^{\prime} / A^{\prime}-P^{\prime}$ ratio represents the proportion of the anterior base (from anterior teeth to first molar) within the entire maxillary dental arch. Higher ratio indicates shorter posterior region and potential need for space. In our study, when these measurements were analyzed, we observed that $A^{\prime}-P^{\prime}$ distance was shorter and $A^{\prime}-6^{\prime} / A^{\prime}-P^{\prime}$ ratio was higher in individuals with open bite. This indicates that posterior arch length deficiency, particularly at the distal of the first molars in anteroposterior dimension, can indeed be present in individuals with open bite. According to Sato and Suzuki (25), one of the reasons for superiority of DFA is that it can facilitate differential diagnosis and guide the decision to perform tooth extraction in cases of tooth-to-denture base discrepancy. Similarly, Celar et al. (15) stated that this ratio can be used to determine whether to perform molar distalization or tooth extraction to resolve tooth crowding. Kim (26) reported that in patients with steepened mandibular and palatal planes and relatively reduced posterior lower facial height, the molars become mesially inclined and their contacts prevent the contact of the anterior teeth. They stated that tooth straightening and/ or extraction is required to eliminate this blockage. According to DFA, molars are the most stable centric stops when forces are applied vertically. However, in cases of posterior crowding, mesial tipping of the molars is observed. This tipping causes the angle between the molars to decrease $(10,11)$. In accordance with these studies, our evaluation demonstrated smaller intermolar angle in the open bite group compared to the control group.
Other dentoalveolar measurements that were used in our study and indicate incisor position were also based on DFA. Accordingly, we assessed the distance and angle of the maxillary and mandibular incisors to the $A B$ line. The measurements revealed only protrusion of the Imandibular incisors in the open bite group compared to the control group. However, an important point to consider regarding these measurements is that the results may be affected by the positions of skeletal points $A$ and $B$. In individuals with open bite, it must be kept in mind that with the posterior rotation of the mandible, point $B$ may be positioned more posteriorly, which may give the impression of mandibular incisor protrusion. We believe the measurements that evaluate tooth positions independent from skeletal variations may be more useful than the dental measurements performed in this analysis.

\section{CONCLUSION}

Null hypothesis was rejected. Our results suggest that there may be a close association between OP inclination and mandibular position in individuals with open bite, and that open bite may arise due to maxillary denture base deficiency, particularly in the posterior region.

Accordingly, DFA can be useful in the differential diagnosis of open bite and in treatment planning, particularly when determining the need for tooth extraction. However, drawbacks such as lack of soft tissue visualization and inadequacy in determining tooth positions require DFA to be used in combination with other analyses.

Ethics Committee Approval: EThis study was approved by Ethics committee of İstanbul Medipol University (Approval No: 639).

Informed Consent: Verbal informed consent was obtained from the patients.

Peer-review: Externally peer-reviewed.

Author Contributions: Design - E.C., A.K.; Supervision - A.K.; Resources - E.C., A.K.; Materials - E.C., A.K.; Data Collection and/or Processing E.C.; Analysis and/or Interpretation - E.C.; Literature Search - E.C.; Writing Manuscript - E.C.; Critical Review - A.K.

Conflict of Interest: The authors have no conflict of interest to declare.

Financial Disclosure: The authors declared that this study has received no financial support.

\section{REFERENCES}

1. Björk A. Variations in the growth pattern of the human mandible: longitudinal radiographic study by the implant method. J Dent Res 1963; 42: 400-11. [Crossref]

2. McNamara JA Jr, Carlson DS. Quantitative anaysis of temporomandibular joint adaptation to protrusive function. Am J Orthod 1979; 76: 593-611. [Crossref]

3. Tanaka ME, Sato S. Longitudinal alteration of the occlusal plane and development of different dentoskeletal frames during growth. Am J Orthod Dentofac Orthop 2008; 134: 602.e1-11. [Crossref] 
4. Kim Jl, Hiyama T, Akimoto S, Shinji H, Tanaka EM, Sato S. Longitudinal study regarding relationship among vertical dimension of occlusion, cant of occlusal plane and antero-posterior occlusal relation. Bull Kanagawa Dent Coll 2006; 34: 130-2.

5. Moss ML. The primacy of functional matrices in orofacial growth. Dent Pract Dent Rec 1968; 19: 65-73.

6. Moss ML, Rankow RM. The role of the functional matrix in mandibular growth. Angle Orthod 1968; 38: 95-103.

7. Moss ML, Salentijn L. The primary role of functional matrices in facial growth. Am J Orthod 1969; 55: 566-77. [Crossref]

8. Petrovic A. Control of postnatal growth of secondary cartilages of the mandible by mechanisms regulating occlusion. Cybernetic model. Trans Eur Orthod Soc 1974: 69-75.

9. Slavicek R. The Masticatory Organ. 1st ed. Klosterneuburg (Austria): GAMMA Medizinisch-wissenschaftliche Fortbildung-AG; 2002.

10. Sato S. Alteration of occlusal plane due to posterior discrepancy related to development of malocclusion. Introduction to Denture Frame Analysis. Bull Kanagawa Dent Col 1987; 15: 115-23.

11. Sato S, Suzuki N, Suzuki Y. Longitudinal study of the cant of the occlusal plane and the denture frame in cases with congenitally missing third molars. Further evidence for the occlusal plane change related to the posterior discrepancy. Nihon Kyosei Shika Gakkai Zasshi 1988; 47:517-25.

12. Schudy FF. Cant of occlusal plane and axial inclinations of teeth. Angle Orthod 1963; 33: 69-82.

13. Sassouni VA. A classification of skeletal facial types. Am J Orthod 1969; 55: 109-23. [Crossref]

14. Moss ML. Beyond roentgenographic cephalometry--what? Am J Orthod 1983; 84: 77-9. [Crossref]

15. Celar AG, Freudenthaler JW, Celar RM, Jonke E, Schneider B. The denture frame analysis: an additional diagnostic tool. Eur J Orthod 1998; 20: 579-87. [Crossref]
16. Kayasu T, Köklü A. Denture Frame Analizi'nin Toplumumuzdaki Normlari Ve Vertikal Yön Etkinliği. Ankara Üniversitesi Sağlık Bilimleri Enstitüsü, Doktora Tezi. 2012.

17. Helm S, Nielsen SS, Skieller V, Björk A. Skeletal maturation of the hand in relation to maximum puberal growth in body height. Tandlaegebladet 1971; 75: 1223-34.

18. Greulich WW, Pyle SI. Radiographic atlas of skeletal development of hand and wrist. 2nd ed. Stanford: Stanford University Press; 1959. [Crossref]

19. Sato S, Sasaguri K, Kamoi S, Goto M, Suzuki Y. Importance of posterior tooth-to-denture base discrepancy in the development of skeletal open bite malocclusion. Nihon Kyosei Shika Gakkai Zasshi 1990; 49: 322-30.

20. Fushima K, Kitamura Y, Mita H, Sato S, Suzuki Y, Kim YH. Significance of the cant of the posterior occlusal plane in class II division 1 malocclusions. Eur J Orthod 1996; 18: 27-40. [Crossref]

21. Uzel I, Enacar A. Ortodontide Sefalometri. 2nd ed. Adana: Çukurova Üniversitesi Basımevi; 2000.

22. Nahoum HI. Vertical proportions and palatal plane in anterior openbite. Am J Orthod 1971; 59: 273-82. [Crossref]

23. English JD, Olfert KDG. Masticatory muscle exercise as an adjunctive treatment for open bite malocclusions. Semin Orthod 2005; 11 : 164-9. [Crossref]

24. Petrovic A. Experimental and cybernetic approaches to the mechanism of action of functional appliances on mandibular growth. McNamara, JA Jr, Ribbens KA, editors. Malocclusion and the periodontium. Ann Arbor: Monograph 15, Craniofacial Growth Series. Center for Human Growth and Development, USA: University of Michigan; 1984.p.213-68.

25. Sato S, Suzuki Y. Relationship between the development of skeletal mesio-occlusion and posterior tooth-to-denture base discrepancy. Nihon Kyosei Shika Gakkai Zasshi 1988; 47: 796-810.

26. Kim YH. Anterior open-bite and its treatment with multiloop edgewise archwire. Angle Orthod 1987; 57: 290-321. 